\title{
Majorana tunneling entropy
}

\author{
Sergey Smirnov* \\ Institut für Theoretische Physik, Universität Regensburg, D-93040 Regensburg, Germany
}

(Received 5 July 2015; revised manuscript received 10 November 2015; published 30 November 2015)

\begin{abstract}
In thermodynamics a macroscopic state of a system results from a number of its microscopic states. This number is given by the exponent of the system's entropy $\exp (S)$. In noninteracting systems with discrete energy spectra, such as large scale quantum dots, $S$ as a function of the temperature has usually a plateau shape with integer values of $\exp (S)$ on these plateaus. Plateaus with noninteger values of $\exp (S)$ are fundamentally forbidden and would be thermodynamically infeasible. Here we investigate the entropy of a noninteracting quantum dot coupled via tunneling to normal metals with continuum spectra as well as to topological superconductors. We show that the entropy may have noninteger plateaus if the topological superconductors support weakly overlapping Majorana bound states. This brings a fundamental change in the thermodynamics of the quantum dot whose specific heat $c_{V}$ acquires low-temperature Majorana peaks which should be absent according to the conventional thermodynamics. We also provide a fundamental thermodynamic understanding of the transport properties, such as the linear conductance. In general our results show that the thermodynamics of systems coupled to Majorana modes represents a fundamental physical interest with diverse applications depending on versatility of possible coupling mechanisms.
\end{abstract}

DOI: 10.1103/PhysRevB.92.195312

PACS number(s): 74.45.+c, 74.25.Bt, 65.40.gd, 74.78.Na

\section{INTRODUCTION}

Majorana fermions [1], particles identified with their own antiparticles, have recently received considerable attention in condensed-matter physics after the seminal Kitaev proposal [2] of a spinless chain model which could be realized using a topological superconducting state especially in mesoscopic setups $[3,4]$.

In particular, topological insulators [5,6] in combination with $s$-wave superconductors are natural candidates for a practical implementation [7,8] of Kitaev's model because the edge states in topological insulators provide a single pair of the Fermi points.

Another practical implementation $[9,10]$ of Kitaev's model is based on spin-orbit-coupled one-dimensional quantum wires placed in an external magnetic field freezing out one spin component. When this effectively "spinless" system is proximity coupled to an $s$-wave superconductor, the one-dimensional wire becomes a topological superconductor implementing Kitaev's model.

As soon as the the Majorana bound states are created there appears the question of how to experimentally detect them. Since these are zero energy modes of a mesoscopic system, it is natural to try to detect them by applying a bias voltage to this mesoscopic system and measuring the current flowing through it. The differential conductance then should have a maximum at zero bias. This has been done in Ref. [11] and signatures of the presence of the Majorana fermions in the system have been reported.

An alternative possibility to detect Majorana fermions is to couple the system supporting Majorana bound states to a normal system via tunneling. The Majorana fermions are then entangled with normal fermions changing drastically the behavior of the initially normal system. This approach was used in Ref. [12] where the normal system was a noninteracting

*sergey.smirnov@physik.uni-regensburg.de quantum dot as well as in Refs. [13-15] where the normal system was a quantum dot with interactions. In both interacting and noninteracting cases the emphasis was made on the transport properties of the quantum dots coupled to topological superconductors. The differential conductance was calculated and the zero bias Majorana peak was found. In the interacting case an interplay between the Majorana and Kondo physics was investigated calculating in addition to the current also the shot noise which is the current-current correlation.

Below we adhere to that alternative strategy but instead of the transport properties of a system coupled to a topological superconductor (or to several topological superconductors) we focus on the thermodynamics of this system and calculate its entropy $S$ and specific heat $c_{V}$. We demonstrate the following.

(1) $S$ as a function of the temperature has a plateau shape with integer values of $\exp (S)$ on these plateaus in the absence of the topological superconductor or when the two Majorana modes within each topological superconductor strongly overlap.

(2) However, $S$ acquires additional plateaus with noninteger values of $\exp (S)$ when only one Majorana mode from each topological superconductor is entangled via tunneling mechanisms with the normal fermions in the quantum dot.

(3) These additional plateaus excite in the temperature dependence of $c_{V}$ additional Majorana peaks being its first low-temperature peaks.

Crucially, as it will become obvious from the text below, this alternative approach is very attractive from the point of view of experimental thermodynamic signatures of Majorana fermions because the quantum dot can be viewed just as a model of a low-energy spectrum of an arbitrary finite (discrete energy spectrum) macroscopic system coupled via tunneling mechanisms to topological superconductors. Therefore, our results are quite general and simple to detect in modern experiments. This is in contrast to setups [16-18] where the Majorana thermodynamics requires specific Kondo spin-flip correlations with the Hamiltonian parameters fine tuned, which is difficult to implement in real experiments; setups with 
more ingredients such as the Josephson vortex dynamics [19], which complicates the experimental observation of the Majorana physics; and setups with only continuum spectra [20], where the entropy as a function of the temperature does not have a plateau shape and, therefore, it is impossible in a realistic experiment (always performed at finite temperatures) to disentangle the Majorana and normal fermion contributions to the entropy.

The paper is organized as follows. In Sec. II we discuss a setup with Majorana tunneling processes involved and calculate its entropy. The thermodynamic results are presented and analyzed in Sec. III. With Sec. IV we conclude the paper.

\section{MAJORANA THERMODYNAMICS}

The system under investigation is shown in Fig. 1. The quantum dot represents a system with a discrete energy spectrum. In the present study we consider a noninteracting quantum dot and, therefore, its Hamiltonian has the form $\hat{H}_{\mathrm{QD}}=$ $\sum_{\alpha, \alpha^{\prime}} h_{\alpha \alpha^{\prime}} d_{\alpha}^{\dagger} d_{\alpha^{\prime}}$, where $\alpha$ is a single-particle index. The quantum dot is coupled to a number (two in the figure but can be arbitrary) of normal metallic contacts with continuum spectra via normal tunneling $\hat{H}_{\mathrm{CD}}=\sum_{l=\mathrm{L}, \mathrm{R}} \sum_{k_{l}, \alpha} c_{l k_{l}}^{\dagger} T_{l k_{l}, \alpha} d_{\alpha}+$ H.c., where $k_{l}$ is the set of quantum numbers characterizing the contact with the number $l$. The contacts are assumed to be noninteracting, $\hat{H}_{\mathrm{C}}=\sum_{l=\mathrm{L}, \mathrm{R}} \sum_{k_{l}} \epsilon_{l k_{l}} c_{l k_{l}}^{\dagger} c_{l k_{l}}$. In addition to the normal tunneling the quantum dot is coupled via another tunneling mechanism to a topological superconductor characterized by the two Majorana modes $\gamma_{j}, \gamma_{j}^{\dagger}=\gamma_{j}, \gamma_{j}^{2}=1$, $j=1,2$. This Majorana tunneling [21] involves only one Majorana mode $\gamma_{1}$, and has the form $\hat{H}_{\mathrm{DM}}=\sqrt{2} \sum_{\alpha} \eta_{\alpha}^{*} d_{\alpha}^{\dagger} \gamma_{1}+$ H.c.. The effective low-energy Hamiltonian of the topological



FIG. 1. (Color online) Schematic picture of the setup. The quantum dot here represents a noninteracting system with a discrete energy spectrum. It is coupled via tunneling junctions to left and right normal metallic contacts (in general the number of normal contacts may be arbitrary) whose energy spectra are continuum. The strength of this tunneling coupling is characterized by the energies $\Gamma_{\mathrm{L}, \mathrm{R}}$ (see the text for the details). The quantum dot is coupled to a topological superconductor whose ends support two Majorana bound states described by the second quantized operators $\gamma_{1,2}$. This tunneling coupling is realized via only one Majorana mode $\gamma_{1}$ and is characterized by the energy $\eta$ (see the text for the details). superconductor is given in terms of the two Majorana modes, $\hat{H}_{\mathrm{TS}}=i \xi \gamma_{2} \gamma_{1}$, where $\xi$ is the energy characterizing the overlap strength [3] of the two Majorana bound states. This implies, similar to many other works on Majorana physics (see, e.g., Refs. [13] and [21]), that we consider energy scales (temperature, tunneling rates, etc.) much smaller than the superconducting gap $\Delta$.

To find the entropy of the quantum dot we construct the field integral in imaginary time [22] for the total partition function $Z_{\text {tot }}$, transform it into a skew-symmetric form, integrate out all the fermionic degrees of freedom, compute the Pfaffian of the matrix of the imaginary time action, and obtain the quantum dot partition function as $Z=Z_{\text {tot }} / Z_{C}$, where $Z_{C}$ is the partition function of the normal metallic contacts.

For the thermodynamic potential $\Omega=-\ln Z / \beta$ we then obtain

$$
\Omega=\Omega_{\mathrm{TS}}+\frac{1}{\beta} \sum_{n \geqslant 0} \ln \operatorname{det}\left[\mathcal{G}_{i \alpha, i^{\prime} \alpha^{\prime}}\left(\omega_{n}\right)\right],
$$

where $\Omega_{\mathrm{TS}}$ is the thermodynamic potential of the isolated topological superconductor, $\beta$ is the inverse temperature, $\beta \equiv 1 / T$ (we use the energy units for the temperature $T$, i.e., $\left.k_{\mathrm{B}}=1\right)$, and $\mathcal{G}_{i \alpha, i^{\prime} \alpha^{\prime}}\left(\omega_{n}\right)$ are the Fourier transforms of the quantum dot imaginary time Green's functions, $\mathcal{G}\left(i \alpha \tau \mid i^{\prime} \alpha^{\prime} \tau^{\prime}\right) \equiv$ $\left\langle\mathrm{T} d_{i \alpha}(\tau) d_{i^{\prime} \alpha^{\prime}}\left(\tau^{\prime}\right)\right\rangle$ [here $i=p, h ; d_{p \alpha}(\tau) \equiv d_{\alpha}^{\dagger}(\tau), d_{h \alpha}(\tau) \equiv$ $d_{\alpha}(\tau)$; and the angular brackets stand for the thermal average], taken at the discrete Matsubara fermionic frequencies $\omega_{n} \equiv$ $\pi(2 n+1) / \hbar \beta$.

Choosing the the single-particle states $|\alpha\rangle$ such that $h_{\alpha \alpha^{\prime}}=$ $\delta_{\alpha \alpha^{\prime}} \epsilon_{\alpha}$ the imaginary time Green's functions become diagonal and Eq. (1) reduces to

$$
\begin{aligned}
\Omega= & \Omega_{\mathrm{TS}}+\frac{1}{2 \beta} \sum_{\alpha, n} \ln \left[\mathcal{G}_{\alpha h p}^{*}\left(\omega_{n}\right) \mathcal{G}_{\alpha h p}\left(\omega_{n}\right)\right. \\
& \left.-\mathcal{G}_{\alpha p p}^{*}\left(\omega_{n}\right) \mathcal{G}_{\alpha p p}\left(\omega_{n}\right)\right] .
\end{aligned}
$$

From Eq. (2) we find the entropy $S=-\partial \Omega / \partial T$ :

$$
\begin{aligned}
S= & \ln \left[\cosh \left(\frac{\xi}{2 T}\right)\right]-\frac{\xi}{2 T} \tanh \left(\frac{\xi}{2 T}\right)+\ln (2) \\
& +\frac{1}{16 \pi i T^{2}} \sum_{\alpha} \int_{-\infty}^{\infty} d \epsilon \frac{\epsilon}{\cosh ^{2}\left(\frac{\epsilon}{2 T}\right)} \ln \left[G_{\alpha}(\epsilon)\right],
\end{aligned}
$$

where

$$
G_{\alpha}(\epsilon) \equiv \frac{G_{\alpha h p}^{A}(-\epsilon) G_{\alpha h p}^{R}(\epsilon)-G_{\alpha h h}^{A}(-\epsilon) G_{\alpha p p}^{R}(\epsilon)}{G_{\alpha h p}^{R}(-\epsilon) G_{\alpha h p}^{A}(\epsilon)-G_{\alpha h h}^{R}(-\epsilon) G_{\alpha p p}^{A}(\epsilon)} .
$$

In Eq. (4) $G_{\alpha i i^{\prime}}^{R, A}(\epsilon)$ are the Fourier transforms of the diagonal elements of the quantum dot real time retarded and advanced Green's functions, $i G^{R, A}\left(i \alpha t \mid i^{\prime} \alpha^{\prime} t^{\prime}\right) \equiv \pm \Theta( \pm t \mp$ $\left.t^{\prime}\right)\left\langle\left\{d_{i \alpha}(t), d_{i^{\prime} \alpha^{\prime}}\left(t^{\prime}\right)\right\}\right\rangle$.

We now apply the general Eqs. (3) and (4) to the case when the quantum dot has a single energy level $\epsilon_{d}$. The normal fermions in the quantum dot are characterized by the spin index, $|\alpha\rangle=|\sigma\rangle$, and, as in Ref. [14], we assume that only one spin component couples to the normal metallic contacts and topological superconductor $\left(\eta_{\sigma}\right.$ is zero for one spin component and is equal to $\eta$ for the opposite one). The single-particle index and summation over it are then irrelevant in Eq. (3). 
To find the retarded and advanced Green's functions we construct the Keldysh field integral [22], perform the Keldysh rotation to get the the retarded-advanced structure of the Keldysh action, integrate out the fermionic degrees of freedom of the normal metallic contacts and topological superconductor, and take the retarded and advanced elements in the inverse matrix of the Keldysh effective action.

For simplicity we assume that all the normal contacts are described by the same quantum numbers $k=\{r, \sigma\}$ ( $r$ is the orbital part and $\sigma$ is the spin part) and are characterized by a constant density of states $v_{\mathrm{C}}$. Additionally, to simplify the calculations we assume that $T_{l r \sigma, \sigma^{\prime}}=\delta_{\sigma \sigma^{\prime}} T_{l \sigma}\left(T_{l \sigma}\right.$ is zero for the same spin component for which $\eta_{\sigma}$ is zero as mentioned above). We then obtain

$$
\begin{aligned}
G_{h p}^{R}(\epsilon)= & \frac{2 \hbar\left\{-4|\eta|^{2} \epsilon-\left(\xi^{2}-\epsilon^{2}\right)\left[i \Gamma+2\left(\epsilon_{d}+\epsilon\right)\right]\right\}}{f(\epsilon)}, \\
G_{p p}^{R}(\epsilon)= & \frac{-8 \hbar \eta^{2} \epsilon}{f(\epsilon)}, \quad G_{h h}^{R}(\epsilon)=\frac{-8 \hbar\left(\eta^{*}\right)^{2} \epsilon}{f(\epsilon)} \\
f(\epsilon)= & \left(\Gamma^{2}+4 \epsilon_{d}^{2}\right) \xi^{2}-4 i \Gamma \epsilon\left(2|\eta|^{2}+\xi^{2}\right) \\
& -\epsilon^{2}\left[16|\eta|^{2}+\Gamma^{2}+4\left(\epsilon_{d}^{2}+\xi^{2}\right)\right]+4 i \Gamma \epsilon^{3}+4 \epsilon^{4}
\end{aligned}
$$

and $G_{h p}^{A}(\epsilon)=\left[G_{h p}^{R}(\epsilon)\right]^{*}, \quad G_{p p}^{A}(\epsilon)=\left[G_{h h}^{R}(\epsilon)\right]^{*}, \quad G_{h h}^{A}(\epsilon)=$ $\left[G_{p p}^{R}(\epsilon)\right]^{*}$. In Eq. (5) $\Gamma \equiv \Gamma_{\mathrm{L}}+\Gamma_{\mathrm{R}}$ and $\Gamma_{l} \equiv \pi v_{C}\left|T_{l}\right|^{2}$.

Using Eqs. (3)-(5) one can calculate the entropy of the quantum dot:

$$
\begin{aligned}
S= & \ln \left[\cosh \left(\frac{\xi}{2 T}\right)\right]-\frac{\xi}{2 T} \tanh \left(\frac{\xi}{2 T}\right)+\ln (2) \\
& +\frac{1}{8 \pi T^{2}} \int_{-\infty}^{\infty} d \epsilon \frac{\epsilon \phi(\epsilon)}{\cosh ^{2}\left(\frac{\epsilon}{2 T}\right)},
\end{aligned}
$$

where $\phi(\epsilon)$ is the phase of the following complex function:

$$
G_{h p}^{A}(-\epsilon) G_{h p}^{R}(\epsilon)-G_{h h}^{A}(-\epsilon) G_{p p}^{R}(\epsilon)=\rho(\epsilon) e^{i \phi(\epsilon)} .
$$

\section{RESULTS AND DISCUSSION}

As it is known from statistical physics [23], a macroscopic state of a system is a collective result of a certain number of its microscopic states. This number is provided by the system's entropy $S$ and is equal to $\exp (S)$. When the temperature is increased, more and more high-energy states will be involved in the macroscopic state and the entropy will grow. If the system's spectrum is discrete and the distance between the levels is large enough, then in certain temperature intervals the entropy will not change, i.e., its temperature dependence will have plateaus on which $\exp (S)$ is an integer showing how many microscopic states are involved in the temperature interval of a given plateau and on the last, the highest, plateau the quantity $\exp (S)$ will provide an integer number equal to the dimensionality of the Hilbert space of the system.

However this standard picture described above changes drastically as soon as the system's normal fermions are entangled with Majorana fermions via a certain mechanism. In the present case this entanglement happens via the tunneling of the strength $\eta$ between the quantum dot and topological superconductor. As shown in Fig. 2, in the absence of the

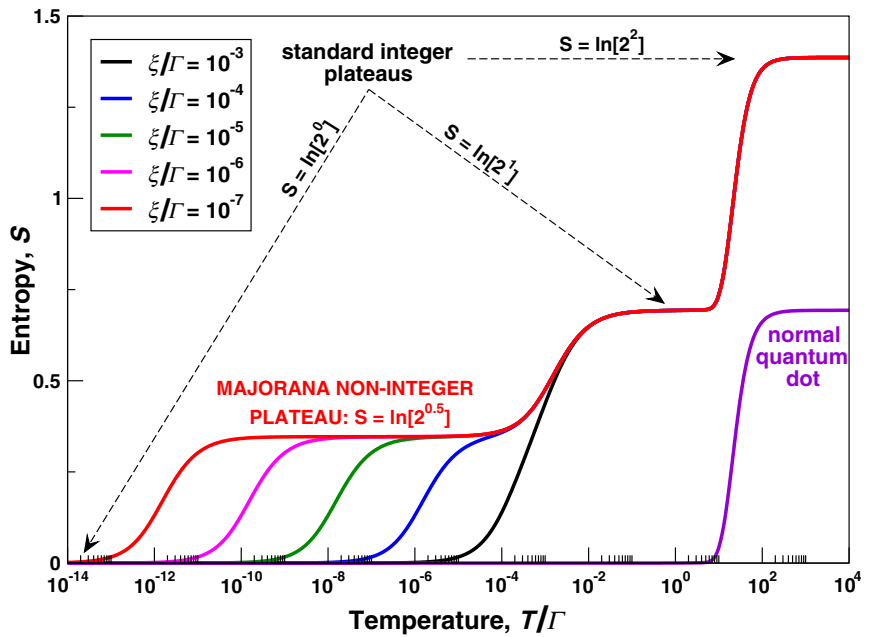

FIG. 2. (Color online) Tunneling entropy $S$ as a function of the temperature $T / \Gamma$ for different values of the overlap energy $\xi / \Gamma$ of the Majorana bound states. The other parameters are $\epsilon_{d} / \Gamma=$ $-50.0, \eta / \Gamma=2.0$. The purple curve shows the tunneling entropy for a normal quantum dot that is the one which is not coupled to any topological superconductor. As expected for a system with a discrete spectrum, $S$ has plateaus. When the overlap of the two Majorana modes is strong, there are only standard plateaus with integer values of $\exp (S)$ similar to normal quantum dots (purple curve). However, when the overlap of the Majorana bound states is weak, there appears an additional plateau with a noninteger value of $\exp (S)$ equal to $\sqrt{2}$.

Majorana fermions (the lowest purple curve) the quantum dot entropy has two standard plateaus with integer values of $\exp (S)$ equal to 1 and 2 corresponding to the two possible states of the quantum dot with one or zero electrons. Similarly, as one can see in Fig. 2, when the quantum dot is coupled to a topological superconductor whose Majorana modes strongly overlap (the black curve, $\xi / \Gamma=10^{-3}$ ) the entropy has plateaus only with integer values of $\exp (S)$. The role of the Majorana fermions in this case is just in the doubling of the dimensionality of the Hilbert space and in the formation of the plateaus with even values of $\exp (S)$. However, when $\xi / \Gamma$ gets smaller and the Majorana bound states overlap weakly there appears a plateau with a noninteger value of $\exp (S)$ which is equal to $\sqrt{2}$. This Majorana plateau represents a signature that the Majorana modes are well separated and strongly entangled with the normal fermions in the quantum dot whose macroscopic state at low temperatures represents now a collective result of the Majorana microscopic states.

To better understand the relevant physics and to see its nontrivial Majorana content the tunneling entropy $S$ might be conceived of three contributions, $S=S_{\mathrm{QD}}+S_{\mathrm{TS}}+S_{\mathrm{T}}$ coming from the quantum dot, $S_{\mathrm{QD}}$, from the topological superconductor, $S_{\mathrm{TS}}$, and from the tunneling interaction between the quantum dot and topological superconductor, $S_{\mathrm{T}}$. There are three characteristic energy scales, $T_{1} \sim \xi^{2}[\xi+$ $\left.\left|\epsilon_{d}\right| \exp \left(-\eta /\left|\epsilon_{d}\right|\right)\right] / \eta^{2}, T_{2} \sim \xi$, and $T_{3} \sim \eta^{2} / 2\left|\epsilon_{d}\right|$ shown in Fig. 3. When $\xi$ decreases, tunneling events involve essentially one Majorana fermion. On one side, during tunneling events the Dirac fermions of the quantum dot transform into the Majorana fermion and vice versa. Such tunneling processes lead to an effective reduction of the number of the fermionic 




FIG. 3. (Color online) The tunneling entropy $S$ (green curve) is the sum of three contributions: the entropy of the quantum dot $S_{\mathrm{QD}}$ (black curve), the entropy of the topological superconductor $S_{\mathrm{TS}}$ (blue curve), and the entropy of the tunneling interaction between the quantum dot and topological superconductor $S_{\mathrm{T}}$ (red curve). The characteristic energies $T_{1}, T_{2}$, and $T_{3}$ (see the main text) indicate the temperature ranges where $S_{\mathrm{T}}$ has the positive and negative plateaus with the absolute value $\ln \left[2^{0.5}\right]$. Here $\xi / \Gamma=10^{-7}$. The other parameters have the same values as in Fig. 2.

degrees of freedom. As a result, $S_{\mathrm{T}}$ is negative in the temperature range from $T_{2}$ to $T_{3}$ (red curve in Fig. 3) leading to a decrease of $S$ in this temperature range from $S=\ln (2)$ (the value it would have had without the tunneling) down to $S=\ln (2) / 2$. On the other side, when $\xi$ is small, the multiple tunneling processes between the quantum dot and topological superconductor give rise to a quantum state where the Dirac fermions of the quantum dot are hybridized with the single Majorana fermion of the topological superconductor in such a way that this new quantum state has a fractional number of degrees of freedom and is responsible for the formation of the entropy plateau $S=\ln (2) / 2$ in the temperature range from $T_{1}$ to $T_{2}$ (red curve in Fig. 3). While it would be reasonable to expect that for this hybridized state the number of degrees of freedom should be between 2 (Dirac fermion) and $\sqrt{2}$ (Majorana fermion), it is surprising that in the present case it is equal to $\sqrt{2}$. In other words, from the point of view of the number of degrees of freedom the hybridized state could have been interpreted as a pure single Majorana fermion. However, what is more important and highly nontrivial is the fact that the both processes, the decrease of the entropy in the temperature range between $T_{2}$ and $T_{3}$ and its increase in the temperature range between $T_{1}$ and $T_{2}$, happen in such a coherent way that there develops a single plateau of the tunneling entropy $S=$ $\ln (2) / 2$ in the temperature range from $T_{1}$ to $T_{3}$. The necessary condition for the validity of our analytical estimate made above for the orders of $T_{1}, T_{2}$, and $T_{3}$ as well as for the formation of a plateau with a noninteger value of $\exp (S)$ is obviously $T_{1} \ll T_{2} \ll T_{3}$, which is the case for small $\xi$ or/and large $\eta$.

From the discussion above it is obvious that the situation with a single plateau on which $\exp (S)$ takes a noninteger value is exceptional because it takes place only when the quantum dot is coupled to just one topological superconductor. Indeed, one might consider a situation when the quantum dot is coupled to $N_{\mathrm{TS}}>1$ topological superconductors. This is experimentally relevant because the quantum dot with a single level $\epsilon_{d}$ represents just a model of the low-energy spectrum of a large macroscopic system whose size is much larger than the relevant size of the topological superconductors and, therefore, this macroscopic system may easily be coupled to many topological superconductors. Since the Majorana modes in different topological superconductors are independent (in contrast to the Majorana modes in the same topological superconductor), their contribution to the tunneling entropy does not depend on the parity of $N_{\mathrm{TS}}$ and, therefore, plateaus with noninteger values of $\exp (S)$ are expected also for any $N_{\text {TS }}>1$. In the case when the overlap energies of all the topological superconductors are approximately the same it is obvious that there will be two Majorana plateaus with $S / \ln (2)=1 / 2\left(\right.$ between $T_{1}$ and $\left.T_{2}\right)$ and $S / \ln (2)=N_{\mathrm{TS}}-1 / 2$ (between $T_{2}$ and $T_{3}$ ). When $N_{\mathrm{TS}}=1, N_{\mathrm{TS}}-1 / 2=1 / 2$ and there is only one Majorana plateau. However, $N_{\mathrm{TS}}-1 / 2>$ $1 / 2$ for $N_{\mathrm{TS}}>1$. The latter case is shown in Figs. 4 and 5 for $N_{\mathrm{TS}}=4$ and 10 , respectively.

The negative contribution to the entropy and the formation of the hybridized state discussed above arise from the tunneling interaction and represent a general mechanism which will take place in other normal systems (not necessarily quantum dots) having tunneling contacts with Majorana systems. The thermodynamic analysis above leads to the following general result. Since in equilibrium the entropy $S$ is maximal, we conclude that the decrease of the entropy $S$ induced by the tunneling interaction in the temperature range between $T_{2}$ and $T_{3}$ has a fundamental consequence that tunneling contacts between normal systems and Majorana systems would eventually vanish in this temperature range and their

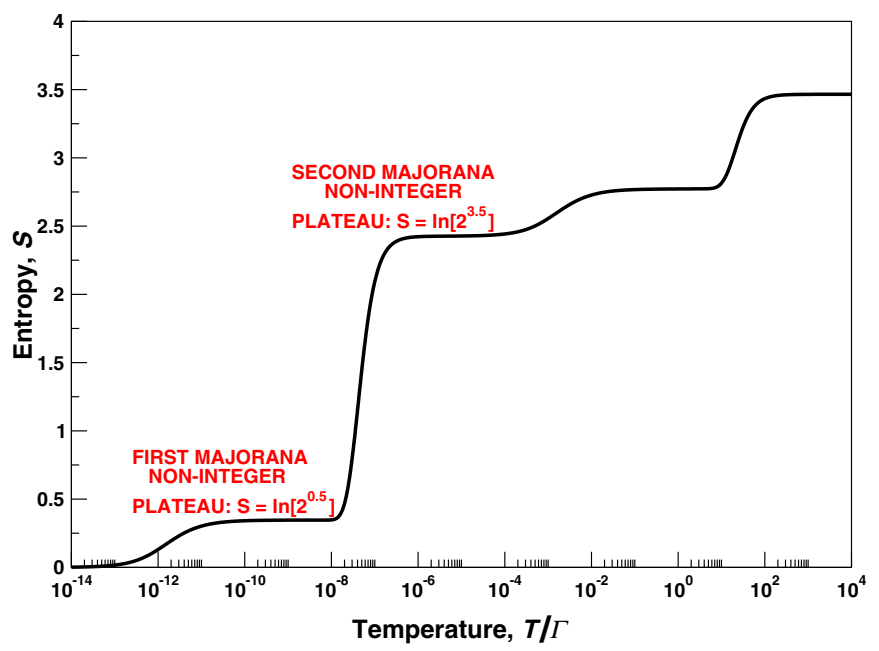

FIG. 4. (Color online) The tunneling entropy $S$ as a function of the temperature $T / \Gamma$ for the case $N_{\mathrm{TS}}=4$. The overlap energies of the topological superconductors are the same, $\xi / \Gamma=10^{-7}$. The other parameters have the same values as in Fig. 2 where now $\eta$ should be understood as the total tunneling strength between the quantum dot and topological superconductors. As expected, the tunneling entropy has two plateaus with noninteger values of $\exp (S)$ equal to $2^{0.5}$ and $2^{3.5}$. 


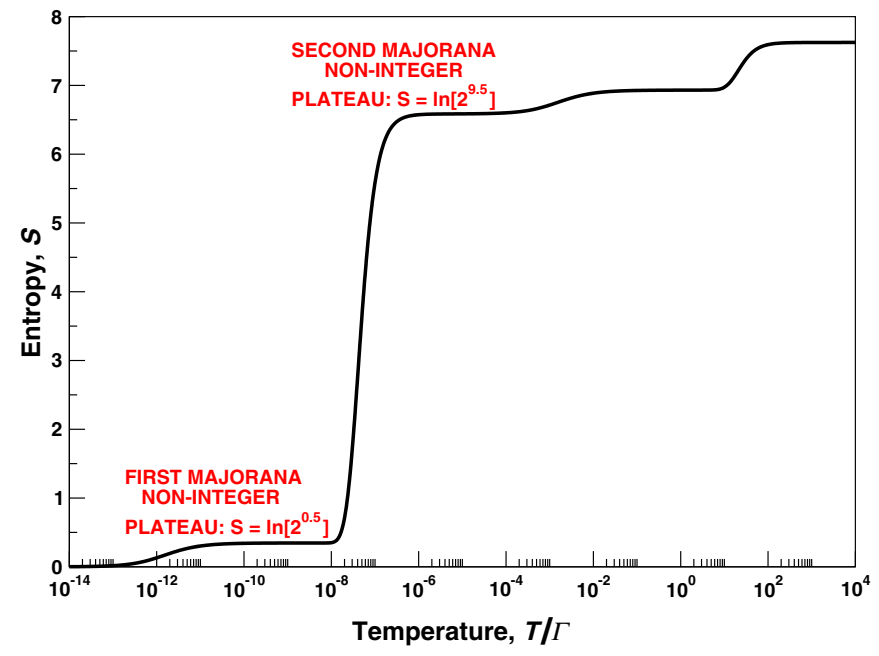

FIG. 5. (Color online) The tunneling entropy $S$ as a function of the temperature $T / \Gamma$ for the case $N_{\mathrm{TS}}=10$. The overlap energies of the topological superconductors are the same, $\xi / \Gamma=10^{-7}$. The other parameters have the same values as in Fig. 2 where now $\eta$ should be understood as the total tunneling strength between the quantum dot and topological superconductors. As expected, the tunneling entropy has two plateaus with noninteger values of $\exp (S)$ equal to $2^{0.5}$ and $2^{9.5}$.

longstanding existence would be possible only due to an external work or a metastable state (which could have a very long lifetime).

We further calculate the specific heat $c_{V}=T \partial S / \partial T$, which is shown in Fig. 6. In the absence of the Majorana fermions,

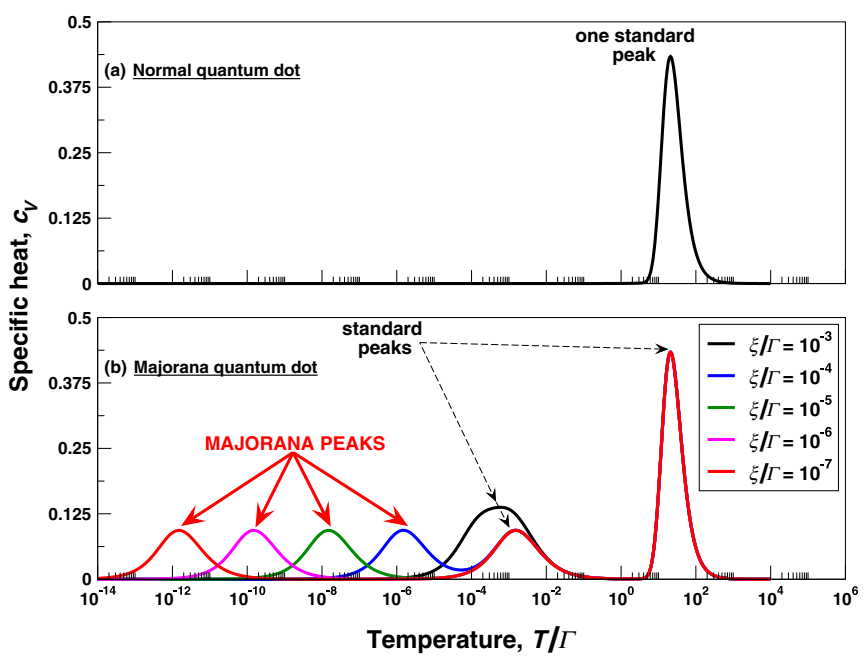

FIG. 6. (Color online) Specific heat $c_{V}$ as a function of the temperature $T / \Gamma$. Part (a) shows the specific heat for a normal quantum dot while part (b) shows it for a Majorana quantum dot with different values of the overlap energy $\xi / \Gamma$ of the Majorana bound states. The other parameters are as in Fig. 2. The plateau shape of the tunneling entropy of a system with a discrete spectrum gives rise to a set of peaks in the specific heat. As the overlap of the Majorana bound states gets weaker, in addition to standard peaks there develops the Majorana leftmost peak which becomes the first low-temperature peak of the specific heat.

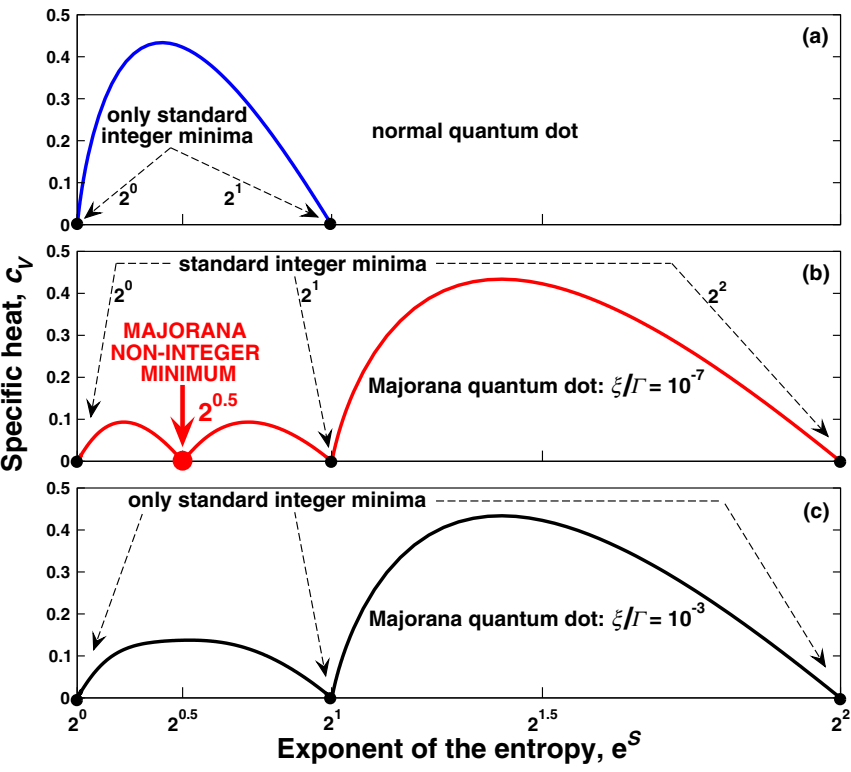

FIG. 7. (Color online) Specific heat $c_{V}$ as a function of the exponent of the tunneling entropy, $\exp (S)$. In this representation the specific heat has the standard minima at integer values. Part (a) shows that for a normal quantum dot these minima are located at $2^{0}$ and $2^{1}$. However, in a quantum dot coupled to a topological superconductor in addition to the standard minima at $2^{0}, 2^{1}$, and $2^{2}$ there appears an additional Majorana noninteger minimum at $2^{0.5}$ as shown in part (b) for weakly overlapping Majorana modes, $\xi / \Gamma=10^{-7}$. When the two Majorana bound states strongly overlap the Majorana noninteger minimum disappears as shown in part (c) for $\xi / \Gamma=10^{-3}$. The other parameters are as in Fig. 2.

Fig. 6(a), the specific heat has a single peak corresponding to the transition between the two integer plateaus of the entropy. This peak remains when the quantum dot is coupled to a topological superconductor, Fig. 6(b). For the case of the strongly overlapping Majorana modes (the black curve) there are two standard peaks corresponding to the transitions between the integer plateaus of the entropy. For the weakly overlapping Majorana bound states there appears the third peak in the specific heat. This Majorana peak is induced by the formation of the Majorana plateau in entropy and represents the first low-temperature peak of the specific heat.

The specific heat is a physical observable which can be experimentally measured. The temperature dependence of the entropy can be recovered from the temperature dependence of $c_{V}$. The specific heat then may be plotted versus the exponent of the entropy, as it is done in Fig. 7. This representation is very instructive. In systems with only normal fermions, Fig. 7(a), the specific heat has standard minima only at integers. However, when the Majorana fermions strongly govern the low-energy physics of the quantum dot, the specific heat acquires an additional Majorana minimum at a noninteger number, Fig. $7(\mathrm{~b})$, equal to $\sqrt{2}$. This noninteger minimum of the specific heat disappears when the two Majorana modes strongly overlap as it is demonstrated in Fig. 7(c).

In spite of the fact that the Majorana plateau is small (of the order of $k_{\mathrm{B}}$ in SI units) it can be recovered from the specific heat already in modern experiments and from values of the 


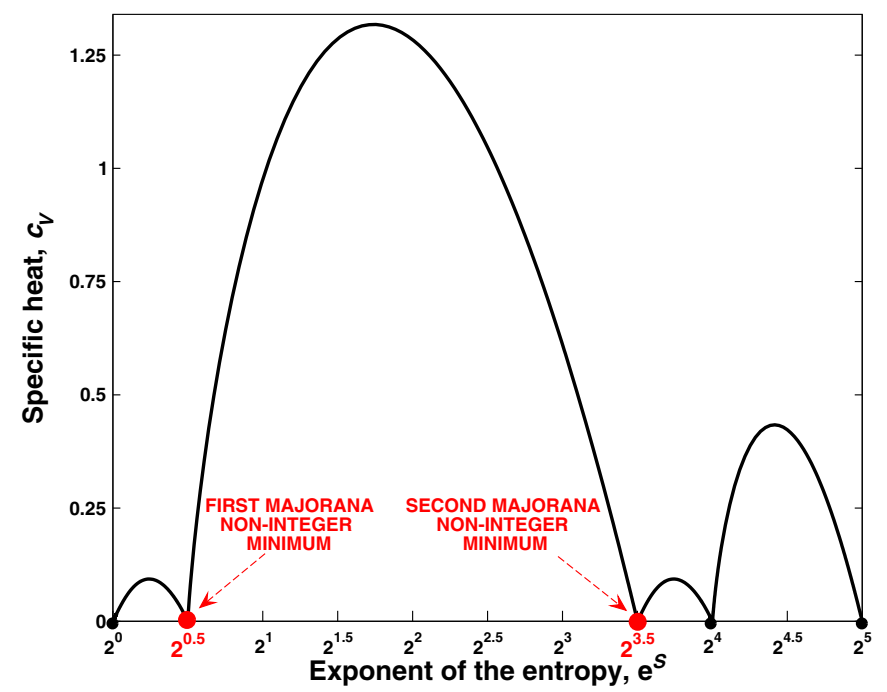

FIG. 8. (Color online) Specific heat $c_{V}$ as a function of the exponent of the tunneling entropy, $\exp (S)$, for the case $N_{\text {TS }}=4$, $\xi / \Gamma=10^{-7}$. The other parameters have the same values as in Fig. 2 where now $\eta$ should be understood as the total tunneling strength between the quantum dot and topological superconductors. As expected, in addition to the standard minima at $2^{0}, 2^{4}$, and $2^{5}$ there appear two additional Majorana noninteger minima at $2^{0.5}$ and $2^{3.5}$.

specific heat which are even smaller than the ones predicted in Fig. 7. Indeed, in experiments of Ref. [24] a very smooth entropy curve below and above $\ln (2) / 2$ is recovered from the experimental measurements of the specific heat which is the quantity used in the integration. This definitely means that already nowadays high precision measurements of $c_{V}$ are possible and may allow us to resolve the Majorana plateau, and one naturally expects that in the near future experiments will become even more precise.

Moreover, the Majorana noninteger entropy plateaus can be experimentally recovered using several topological superconductors $N_{\mathrm{TS}}>1$ as discussed above in connection with Figs. 4 and 5. Indeed, as it is obvious from Figs. 4 and 5, the specific heat coming from the transition between the two noninteger Majorana plateaus will grow with $N_{\text {TS }}$. Therefore, it will be even easier to experimentally detect the corresponding peak in the specific heat and its two noninteger edges as it is shown in Figs. 8 and 9 for $N_{\mathrm{TS}}=4$ and 10 , respectively.

Concerning the temperatures at which the Majorana noninteger plateau can be experimentally observed we find that it may be visible already at $T / \Gamma=5 \times 10^{-3}$ as shown in Fig. 10. This temperature is well within the range of modern experiments. Indeed, for $\Gamma \approx \Delta(\Delta$ is the induced superconducting gap) one obtains $T \approx 1 \mathrm{~K}$ for $\Delta=15 \mathrm{meV}$ from the experiments of Ref. [25]. The temperatures $T \approx 1 \mathrm{~K}$ are easily reachable in modern physical laboratories. Even with $\Delta=250 \mu \mathrm{eV}$ from the experiments of Ref. [11] one gets $T \approx 15 \mathrm{mK}$, which is within modern experiments as well.

Finally, let us consider an application of the thermodynamic analysis to the transport properties. For example, the linear conductance, $\sigma_{L}$, which is a transport property characterized by an equilibrium state of the quantum dot, may be found as

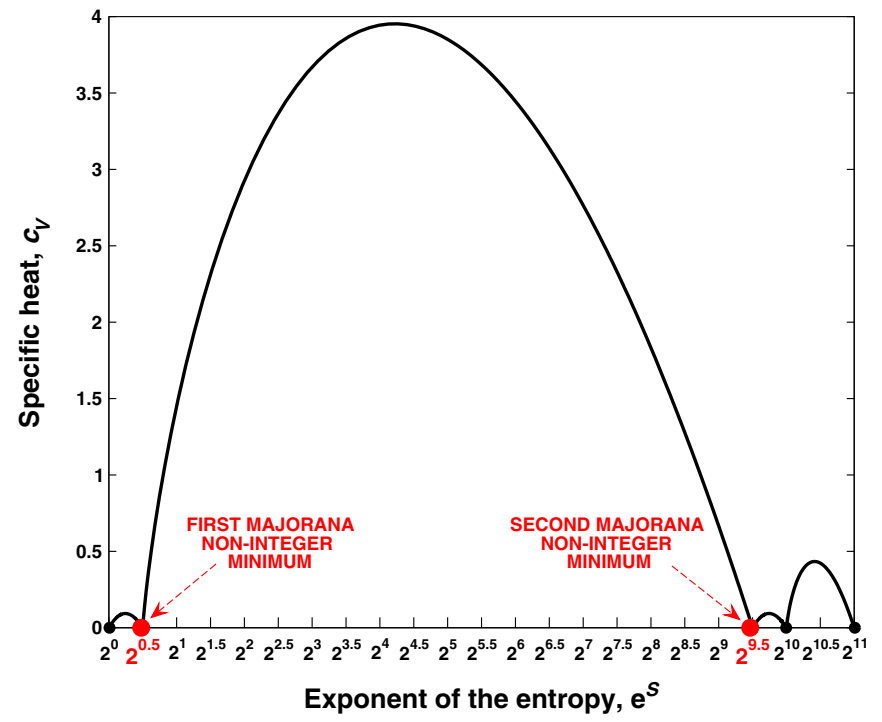

FIG. 9. (Color online) Specific heat $c_{V}$ as a function of the exponent of the tunneling entropy, $\exp (S)$, for the case $N_{\mathrm{TS}}=10$, $\xi / \Gamma=10^{-7}$. The other parameters have the same values as in Fig. 2 where now $\eta$ should be understood as the total tunneling strength between the quantum dot and topological superconductors. As expected, in addition to the standard minima at $2^{0}, 2^{10}$, and $2^{11}$ there appear two additional Majorana noninteger minima at $2^{0.5}$ and $2^{9.5}$.

the derivative $\sigma_{L}=\partial I / \partial V$ of the current,

$$
\begin{aligned}
I & =-\frac{e \Gamma}{4 \pi \hbar^{2}} \int_{-\infty}^{\infty} d \epsilon \operatorname{Im}\left[G_{h p}^{R}(\epsilon)\right]\left[n_{\mathrm{L}}(\epsilon)-n_{\mathrm{R}}(\epsilon)\right], \\
n_{\mathrm{R}, \mathrm{L}}(\epsilon) & =\frac{1}{\exp [\beta(\epsilon \pm e V / 2)]+1},
\end{aligned}
$$

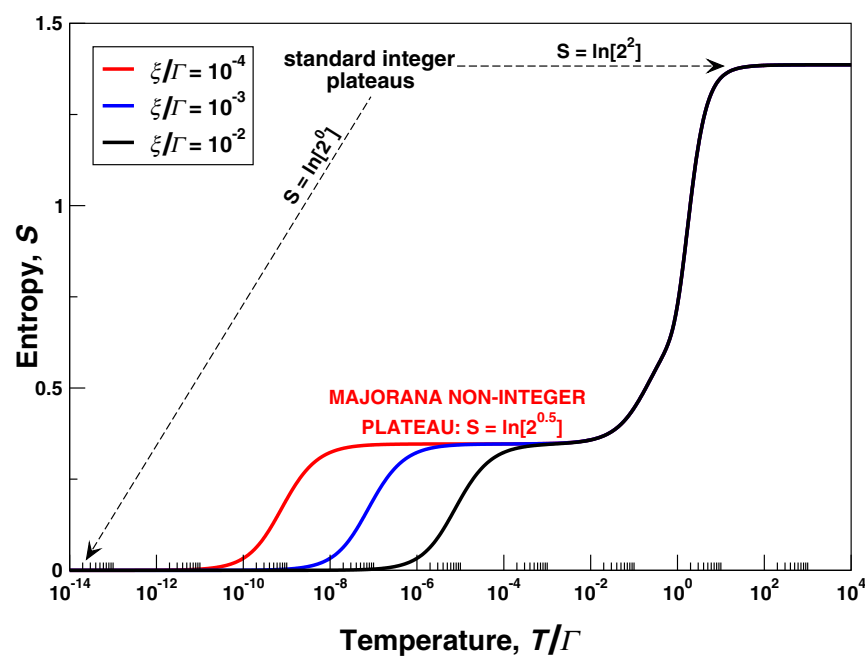

FIG. 10. (Color online) Tunneling entropy $S$ as a function of the temperature $T / \Gamma$ for different values of the overlap energy $\xi / \Gamma$ of the Majorana bound states. The other parameters are $\epsilon_{d} / \Gamma=-1.0$, $\eta / \Gamma=2.0$. The standard integer plateau with $\exp (S)=2^{1}$ is washed out by the significantly expanding noninteger Majorana plateau with $\exp (S)=\sqrt{2}$ which is visible already at $T / \Gamma=5 \times 10^{-3}$. 


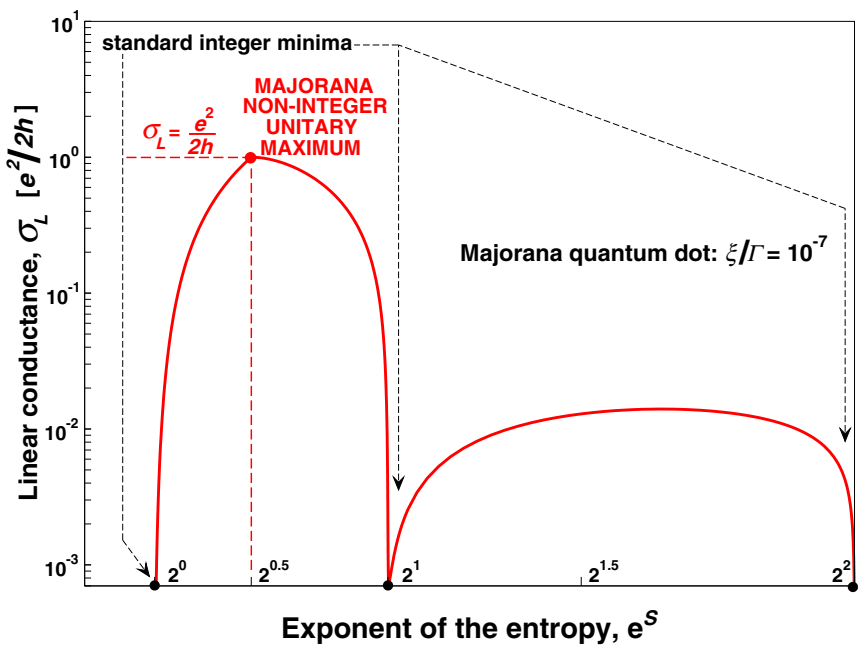

FIG. 11. (Color online) Linear conductance $\sigma_{L}$ as a function of the exponent of the tunneling entropy, $\exp (S)$. In this representation the linear conductance has the standard minima at the integer values $2^{0}, 2^{1}$, and $2^{2}$. The two Majorana modes overlap weakly, $\xi / \Gamma=10^{-7}$, and this results in a Majorana unitary maximum, $\sigma_{L}=e^{2} / 2 h$, at the noninteger value $\sqrt{2}$. The other parameters are as in Fig. 2 .

with respect to the voltage $V$ at $V=0$. In Eq. (8) $e$ is the electronic charge and $n_{\mathrm{R}, \mathrm{L}}(\epsilon)$ are the Fermi-Dirac distributions in the right and left normal metallic contacts and we have assumed $\Gamma_{\mathrm{L}}=\Gamma_{\mathrm{R}}$ for simplicity. When the two Majorana bound states in the topological superconductor overlap weakly the linear conductance at low temperatures reaches the unitary maximum $e^{2} / 2 h$, where $h$ is Planck's constant. In many recent publications [12-15] it is argued that this unitary maximum is a result of the tunneling coupling of the quantum dot to the topological superconductor. Our results provide an alternative thermodynamic explanation based on the structure of the macroscopic state of the quantum dot. As it is shown in Fig. 11, the linear conductance as a function of the exponent of the entropy reaches the Majorana unitary maximum at the noninteger value $\exp (S)=\sqrt{2}$ which corresponds to the Majorana plateau in Fig. 2. This shows that the unitary maximum of the linear conductance is a consequence of the quantum dot macroscopic state composed of a noninteger number of microscopic states, corresponding to the halffermionic value of the entropy, and that the temperature range of the linear conductance unitary maximum coincides with the temperature range of the noninteger Majorana plateau of the tunneling entropy.

\section{CONCLUSION}

To conclude, we would like to emphasize two aspects of our work which are fundamental for the Majorana physics in general but currently not well explored. Our results demonstrate the fundamental role of entropy in the deep physical understanding of macroscopic states of systems coupled to Majorana bound states and, as a result, in the deep physical understanding of the behavior of their observables. Therefore, the thermodynamics of such systems represents on one side an independent research field with its own, thermodynamic, signatures for Majorana fermions, and on the other side it provides a connection to other fields, such as transport, advancing their further understanding on a deep fundamental basis.

\section{ACKNOWLEDGMENT}

Support from the Deutsche Forschungsgemeinschaft under Program No. SFB 689 is acknowledged.
[1] E. Majorana, Nuovo Cimento 14, 171 (1937).

[2] A. Yu. Kitaev, Phys. Usp. 44, 131 (2001).

[3] J. Alicea, Rep. Prog. Phys. 75, 076501 (2012).

[4] K. Flensberg, Semicond. Sci. Technol. 27, 124003 (2012).

[5] M. Z. Hasan and C. L. Kane, Rev. Mod. Phys. 82, 3045 (2010).

[6] X.-L. Qi and S.-C. Zhang, Rev. Mod. Phys. 83, 1057 (2011).

[7] L. Fu and C. L. Kane, Phys. Rev. Lett. 100, 096407 (2008).

[8] L. Fu and C. L. Kane, Phys. Rev. B 79, 161408(R) (2009).

[9] R. M. Lutchyn, J. D. Sau, and S. Das Sarma, Phys. Rev. Lett. 105, 077001 (2010).

[10] Y. Oreg, G. Refael, and F. von Oppen, Phys. Rev. Lett. 105, 177002 (2010).

[11] V. Mourik, K. Zuo, S. M. Frolov, S. R. Plissard, E. P. A. M. Bakkers, and L. P. Kouwenhoven, Science 336, 1003 (2012).

[12] E. Vernek, P. H. Penteado, A. C. Seridonio, and J. C. Egues, Phys. Rev. B 89, 165314 (2014).

[13] M. Cheng, M. Becker, B. Bauer, and R. M. Lutchyn, Phys. Rev. X 4, 031051 (2014).

[14] D. E. Liu, M. Cheng, and R. M. Lutchyn, Phys. Rev. B 91, 081405(R) (2015).
[15] D. A. Ruiz-Tijerina, E. Vernek, L. G. G. V. Dias da Silva, and J. C. Egues, Phys. Rev. B 91, 115435 (2015).

[16] A. M. Tsvelick and P. B. Wiegmann, Z. Phys. B 54, 201 (1984).

[17] I. Affleck and A. W. W. Ludwig, Phys. Rev. Lett. 67, 161 (1991).

[18] V. J. Emery and S. Kivelson, Phys. Rev. B 46, 10812 (1992).

[19] C.-Y. Hou, K. Shtengel, G. Refael, and P. M. Goldbart, New J. Phys. 14, 105005 (2012).

[20] I. Affleck and D. Giuliano, J. Stat. Mech. (2013) P06011.

[21] K. Flensberg, Phys. Rev. B 82, 180516(R) (2010).

[22] A. Altland and B. Simons, Condensed Matter Field Theory, 2nd ed. (Cambridge University, Cambridge, 2010).

[23] L. D. Landau and E. M. Lifshitz, Statistical Physics. Part 1: Course of Theoretical Physics (Pergamon, New York, 1980), Vol. 5.

[24] T. Liang, S. M. Koohpayeh, J. W. Krizan, T. M. McQueen, R. J. Cava, and N. P. Ong, Nature Communications 6, 7611 (2015).

[25] E. Wang, H. Ding, A. V. Fedorov, W. Yao, Z. Li, Y.-F. Lv, K. Zhao, L.-G. Zhang, Z. Xu, J. Schneeloch, R. Zhong, S.-H. Ji, L. Wang, K. He, X. Ma, G. Gu, H. Yao, Q.-K. Xue, X. Chen, and S. Zhou, Nature Physics 9, 621 (2013). 